\title{
Visualization of flow separation inside cut kerf during laser cutting of thick sections
}

\author{
Chi Zhang \\ The State Key Laboratory of Tribology, Tsinghua University, Beijing 100084, China and Department \\ of Mechanical Engineering, Tsinghua University, Beijing 100084, China \\ Peng Wen ${ }^{\text {a) }}$ \\ The State Key Laboratory of Tribology, Tsinghua University, Beijing 100084, China and \\ Department of Mechanical Engineering, Tsinghua University, Beijing 100084, China \\ Zhaohui Yao \\ School of Aerospace, Tsinghua University, Beijing 100084, China \\ Yueming Yuan and Xuejun Fan \\ State Key Laboratory of High Temperature Gas Dynamics, Institute of Mechanics, Chinese Academy \\ of Sciences, Beijing 100190, China
}

(Received 29 February 2016; accepted for publication 3 March 2016; published 31 March 2016)

\begin{abstract}
The behavior of oxygen gas not only determines the oxidation heat input but also affects the removal of molten metal during laser-oxygen cutting. Flow separation inside the cut kerf is the major problem for the deterioration of cut ability and cut quality, especially for cutting thick steel sections, because it significantly weakens the shear stress on molten slag and changes the pressure gradient along the cut front. There have been some researches on flow separation using numerical simulation and model kerf. However, many experimental details and results are unclear, and the numerical simulation is questionable due to lack of experimental verification. In this paper, a model kerf is set up based on the previous results of observation and cutting trials. High resolution and high speed Schlieren visualization is used to observe the formation of flow separation in the model kerf. Stand-off distance, inlet pressure, cut front slope, nozzle displacement, kerf width, and nozzle tilting angle are used as variables for Schlieren visualization of flow separation for both a conical subsonic and a minimum length nozzle supersonic nozzle. The effect of the above parameters on the position and the pattern of flow separation is discussed based on the gas dynamic theory. It is found that nozzle displacement, kerf width, and nozzle tilting angle are the key factors for flow separation. Possible strategies for controlling flow separation are also proposed for both subsonic and supersonic nozzles. @ 2016 Laser Institute of America. [http://dx.doi.org/10.2351/1.4943997]
\end{abstract}

Key words: laser cutting, gas flow, schlieren visualization, cutting nozzle

\section{INTRODUCTION}

Laser cutting has become one of the most common used laser processing technologies in industrial application. Fast speed, good quality, and high flexibility are concluded as the main advantages of laser cutting. ${ }^{1}$ One of the biggest challenges lies in how to cut thick section plates regarding the further expanding application, especially in heavy industry like shipbuilding, nuclear engineering, and marine engineering. ${ }^{2}$ For stainless steel and aluminum thick plates, inert gas laser cutting with a high laser power and a high gas pressure is always necessary. A maximum thickness around 15 to $20 \mathrm{~mm}$ can be reached with a power laser over $4 \mathrm{~kW}$ and nitrogen gas from 5 to 15 bars. $^{3}$ For carbon steel, oxygen laser cutting is often used. Fe-O oxidation reaction not only provides supplies additional combustion heat but also helps to remove the molten metal due to the formation of Fe oxidation products with low melting point and low viscosity. A

\footnotetext{
a) Author to whom correspondence should be addressed; electronic mail: wenpeng@mail.tsinghua.edu.cn
}

maximum thickness around $20 \mathrm{~mm}$ can be reached by conventional oxygen laser cutting with a laser power around $3-4 \mathrm{~kW}$ and oxygen gas from 1 to 3 bars. Thickness around $50 \mathrm{~mm}$ can be cut by LASOX (laser-assisted oxygen cutting) with a $1 \mathrm{~kW}$ laser and oxygen gas about 8 bars. ${ }^{4}$ In LASOX, a defocused laser heats up the surface of cut pieces and initiates $\mathrm{Fe}-\mathrm{O}$ combustion, which provides the heat to melt metal. A supersonic jet flow, whose diameter is less than the laser spot, blows off the molten metal in the cut kerf. Since the continuous $\mathrm{Fe}-\mathrm{O}$ combustion can provide enough heat to melt the metal, theoretically this method has no limit for the cut thickness only if the jet flow can stay straight and strong to remove the molten metal.

As described above, materials are cut in half under the coupling effect of heat and assistant gas during laser cutting. Heat, either laser energy or combustion heat, is necessary to melt the metal; while the momentum of assistant gas is necessary to form the cut kerf. With the increase of cutting thickness, the necessary heat can increase with strong power source or method like LASOX. But the mechanism of dross removal becomes complicated especially when the aspect 
ratio between cut thickness and kerf width is high (more than 20 ) in the inert-gas laser cutting and conventional laseroxygen cutting. Cut quality and ability deteriorates greatly due to the deterioration of gas flow in cut kerf. As a result, it is of great importance to study the dynamic behavior of assistant gas. Regarding dross removal during laser cutting, pressure gradient and shear force on the cut front are the two main factors. ${ }^{5}$ Flow separation on the cut front seems unavoidable during laser cutting thick sections, which is considered as the key problem affecting the efficiency of dross removal, thus cut quality. When flow separation occurs, pressure gradient will change its sign causing a reduction of gas velocity and the detachment of streamlines from the cut front. Shear force on the dross decreases greatly after the separation point. Vortex in the opposite direction of molten removal happens and weakens dross removal. Meanwhile, the vortex entrains air into the cut kerf which causes impurity of oxygen and reduces oxidation efficiency during oxygen laser cutting. The vortex also causes oxidation of cut surface during inert gas laser cutting of stainless steel.

Therefore, it is of great importance to study the characteristics and mechanism of flow separation inside cut kerf during laser cutting especially for thick sections.

Numerical simulation based on computation fluid dynamics is the main tool to study the complex phenomenon of flow separation. It is necessary to carry out experiments to understand the basic mechanism and verify simulation results. Several experimental investigations have been done on flow separation using model kerfs with Schlieren visualization. Brandt ${ }^{6}$ set the model kerf to be $12 \mathrm{~mm}$ high with a constant $1.6 \mathrm{~mm}$ kerf width and the cut front slope to be $12^{\circ}$. It was aimed to represent the approximate melt-front lag known to occur in inert gas laser cutting. The effect of offaxis angle and pressure on flow separation was studied using subsonic nozzle. $\operatorname{Man}^{7}$ set the model kerf to be 10 and $20 \mathrm{~mm}$ high with 0.5 and $0.7 \mathrm{~mm}$ kerfs, and the cut front slope also to be $12^{\circ}$. He studied the effect of inlet pressure, kerf width, kerf depth, and stand-off distance on flow separation for sonic and supersonic nozzles. However, the nozzles were all put with their edges to the cutting front, and the results were not clear enough. Kovalev ${ }^{8}$ set the model kerf to be 10,15 , and $20 \mathrm{~mm}$ with 0.7 and $1.0 \mathrm{~mm}$ kerf, and the cut front slope to be $0^{\circ}$. The setting was said to be based on theoretical analysis and high speed photography of laser cutting of Rose Alloy. He studied the effect of inlet pressure and kerf width on flow separation for sonic and supersonic nozzles. Previous researchers have agreed that flow separation point will move down with increasing inlet pressure and kerf width. The flow separation can be eliminated for a $15 \mathrm{~mm}$ thick kerf with an inlet pressure of 15-20 bars for sonic nozzle and 10-15 bars for supersonic nozzle. However, flow separation in a kerf thicker than $20 \mathrm{~mm}$ is still unsolved. Few reports are found on the experimental investigation of the effect of cut front slope, representing the cutting velocity, and the periodical change of cutting front slope under different cutting parameters. ${ }^{9,10}$ Moreover, it is unclear how the displacement between nozzle axis and cut front affects flow separation, representing different focal point position during laser cutting thick sections. Effect of a kerf wider than $2 \mathrm{~mm}$ representing LASOX process on flow separation has not been studied. Effect of nozzle type on flow separation under different conditions have not been compared and studied thoroughly.

In this paper, it is purposed to understand the effect of main cutting parameters on flow separation, and helps to find ways to inhibits flow separation. Schlieren visualization is used to make a thorough study of the effect of cut front slope, nozzle displacement, and big kerf width on flow separation with model cut kerf. The different characteristics of flow separation are compared and discussed using conical and supersonic nozzles.

\section{EXPERIMENT METHOD AND PARAMETER SETTING}

A model kerf was used to simulate cut kerf during laser cutting as Fig. 1 shows. The model kerf consists of two highpurity quartz glass with a controlled gap between. It was designed geometrically similar to the real cut kerf. The gap depth was set to be $20 \mathrm{~mm}$, simulating laser cutting depth. The gap width between the quartz glass was controlled by aluminum plates with different thickness. Gap width of $1 \mathrm{~mm}$ simulates cut kerf formed in conventional laser cutting, while gap width of 2 and $2.5 \mathrm{~mm}$ for cut kerfs in LASOX cutting. The inclination angle of plate was set to simulate the slope of cut front, and the value was set to be $0^{\circ}, 4^{\circ}, 8^{\circ}$, and $12^{\circ}$. The different slope represents the change of cut front under different cutting velocity during real cutting conditions. Nozzle displacement was set to be 0 and $1 \mathrm{~mm}$, representing different focal point positions. The range of inlet pressure was set to be 3-10 bars. The stand-off distance was set to be $1-3 \mathrm{~mm}$.

A Z-type Schlieren optical system with a halogen light was used to visualize the free jet and the dynamic behavior of gas flow inside the model kerf. The diameter of the main mirror of the system was $150 \mathrm{~mm}$. The parabolic light travels vertically through the quartz (Schlieren Window) to capture the shock structures inside the model kerf during visualization of dynamic behavior inside the model kerf. The pictures are taken by a high speed camera with a resolution of $1280 \times 800$ and exposure time of $300 \mu \mathrm{s}$. A commercial conical nozzle with an exit diameter of $2.5 \mathrm{~mm}$ and a designed Mach 2.0 MLN (Minimum Length Nozzle) supersonic nozzle with a designed pressure of 7 bars, a throat diameter of

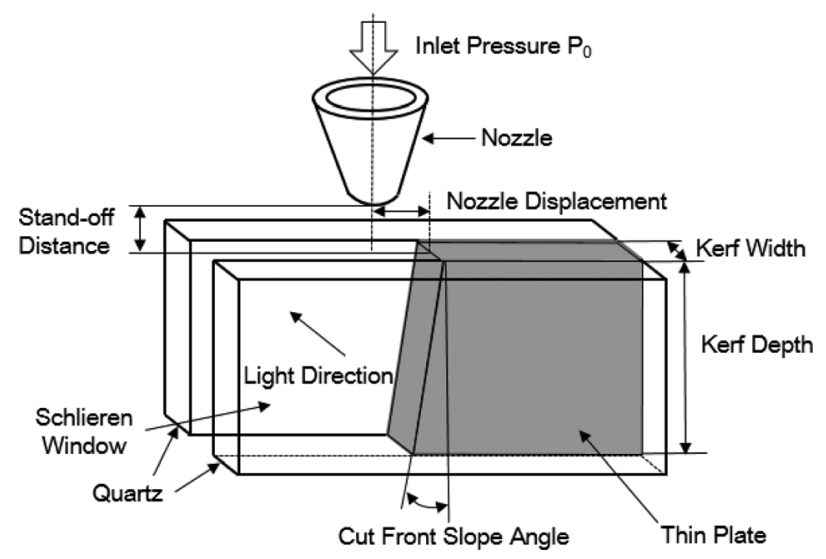

FIG. 1. Schematic diagram of Schlieren visualization inside model kerf. 
$2 \mathrm{~mm}$, and an exit diameter of $2.64 \mathrm{~mm}$ used to compare the nozzle effect on flow separation are shown in Fig. 2.

\section{RESULTS AND DISCUSSION}

\section{A. Free jet visualization}

The Schlieren results of free jet flow with the conical and MLN nozzle under different inlet pressures are shown in Figs. 3(a)-3(f). Figures 3(a)-3(c) show that when the inlet pressure of the conical nozzle is greater than 4 bars, the jet at the nozzle exit is under-expanded. Expansion waves reach the jet boundary and reflect as compression waves. The compression waves become stronger when the inlet pressure is higher and reflect as Mach disk shown in Figs. 3(b) and 3(c). The higher the inlet pressure, the lower and stronger the Mach disk is. Jet exiting from the nozzle expands and accelerates to supersonic first, decelerates to subsonic after passing through the Mach disk and re-accelerates. However, jet passing through a Mach disk suffers from great energy loss and becomes turbulent, which is definitely bad for laser cutting. Figures 3(d)-3(f) show that for the MLN nozzle whose design pressure is 7 bars, when the inlet pressure is 4 bars, the jet at the nozzle exit is over-expanded and is compressed at the nozzle exit; when the inlet pressure is 10 bars, the jet at the nozzle exit is under-expanded and expand from the nozzle exit; when the inlet pressure is 7 bars, the jet is perfectly expanded at the nozzle exit and propagated at a nearly constant velocity exiting from the nozzle. Free jet visualization is a way of evaluating nozzle performance; however, jet structure must be different when the jet enters a narrow kerf at a stand-off distance shorter than the first shock cell under high inlet pressures. Wave interactions between the jet boundary, the kerf walls, and the cut front make the jet dynamic behavior complex inside the cut kerf. It is of great importance to study the jet dynamic behavior in order to improve the cut ability and quality of thick plate laser cutting.

Flow separation is the major dynamic behavior and major problem causing deterioration of cut quality during thick plate laser cutting. It occurs when the mainstream flow decelerates and the static pressure increases in the propagation direction according to Bernoulli's equation. In laser cutting process, the

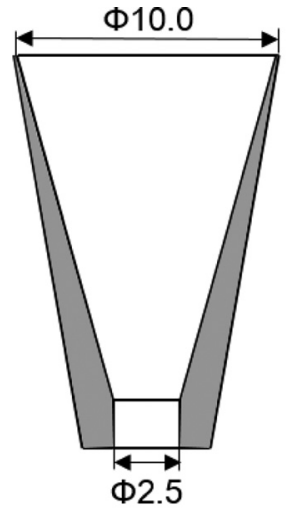

(a)

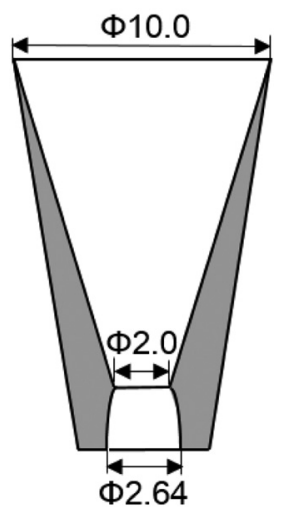

(b)
FIG. 2. Schematic diagram of the two nozzles used in the experiments: (a) conical nozzle and (b) MLN nozzle.

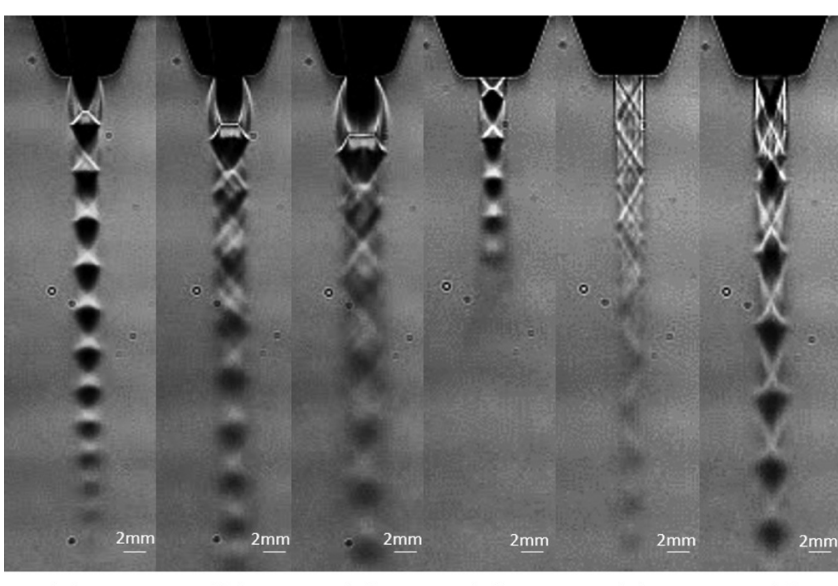
(a)
(b)
(c)
(d)
(e)
(f)

FIG. 3. Free jet Schlieren results under different inlet pressures with different nozzles: (a) conical nozzle: 4 bars, (b) conical nozzle: 7 bars, (c) conical nozzle: 10 bars, (d) MLN nozzle: 4 bars, (e) MLN nozzle: 7 bars, and (f) MLN nozzle: 10 bars.

viscous shear in the boundary layer continuously decelerates flow in the boundary layer especially close to the solid cut front. A deformation of velocity profile is formed. Therefore, at some downstream point located on the cut front, the velocity of the streamlines close to the kerf wall becomes zero and the layer thickness thickens rapidly to satisfy the continuity law. Below the separation point, the fluid near the kerf wall starts moving in the upstream direction pushing the boundary layer and the mainstream flow away from the kerf wall. Thus, the flow separation is formed. Define the distance between the point where the flow detach from the cut front and the upper surface of the cut kerf as the flow separation depth, $\mathrm{D}_{\mathrm{s}}$. The stream begins to become turbulent and vortex appears below the separation point. The detachment of the stream from the cut front strongly weakens the shear stress on the molten slay, and the pressure gradient changes its sign at the separation point, which makes dross removal difficult. Moreover, the vortex accumulates the dross, and the dross moves circling until either be blown out from the channel at a low speed or freeze onto the sidewall generating deep gouging and dross attachment. This explains the low cut quality especially at the lower part of the cut surface during laser cutting thick plates.

\section{B. Flow visualization inside model kerf}

\section{Stand-off distance}

The effect of stand-off distance on the position of flow separation is shown in Fig. 4. Figures 4(a)-4(c) show Schlieren visualization images using a conical nozzle at the inlet pressure of 7 bars. The distance Ds between the separation point and the top surface decreases, and was measured as $4.5,3.7$, and $3.4 \mathrm{~mm}$ when the stand-off distance was 1,2 , and $3 \mathrm{~mm}$. When gas jet enters the kerf, the expansion wave formed inside the kerf reaches gas boundary and is reflected as an incident shock, shown with a white arrow in Fig. 4(a). Jet passing through the incident shock compress and form an adverse pressure gradient causing flow separation from the cut front. As a result, the higher position of the incident shock, the higher the separation point. According to gas dynamic theories, 
the higher the gas velocity, the bigger the reflect angle between the shock and gas boundary, thus the higher position of the incident shock. For the conical nozzle at an inlet pressure of 7 bars, the jet at the nozzle exit is highly under-expanded and will expand and accelerate from the nozzle exit until the location of the first Mach Disk (3.8 mm from nozzle exit measured from Schlieren result of free jet). So when the stand-off distance increases from 1 to $3 \mathrm{~mm}$, the velocity of the jet entering the kerf increases as well. Thus the position of the incident shock is higher and Ds becomes smaller.

Figures 4(d)-4(f) show the results of MLN nozzle at the inlet pressure of 7 bars. Compared with the conic nozzle, Ds stays constant nearly as $4.1 \mathrm{~mm}$ when the stand-off distance changes from 1 to $3 \mathrm{~mm}$. For the designed MLN nozzle at its working pressure 7 bars, the jet at the nozzle exit is nearly perfect-expanded. The velocity keeps constant when the stand-off distance increases from 1 to $3 \mathrm{~mm}$. Thus, the reflect angle also keeps constant and Ds does not change. This also explains the fact that the operating tolerance of stand-off distance is bigger for supersonic nozzles than conical nozzles during laser cutting.

\section{Inlet pressure and cut front slope}

The effect of inlet pressure and cut front slope on the position of flow separation is shown in Fig. 5. The kerf width is fixed to $1 \mathrm{~mm}$. For the conical nozzle in Fig. 5(a), $D_{\mathrm{s}}$ increases with increasing the inlet pressure from 3 to 10 bars for all the four cut front slopes. The longest Ds is about $9 \mathrm{~mm}$ with 10 bars and $4^{\circ}$ slope. Note that it is less than half of the kerf depth $10 \mathrm{~mm}$. For the four different cut front

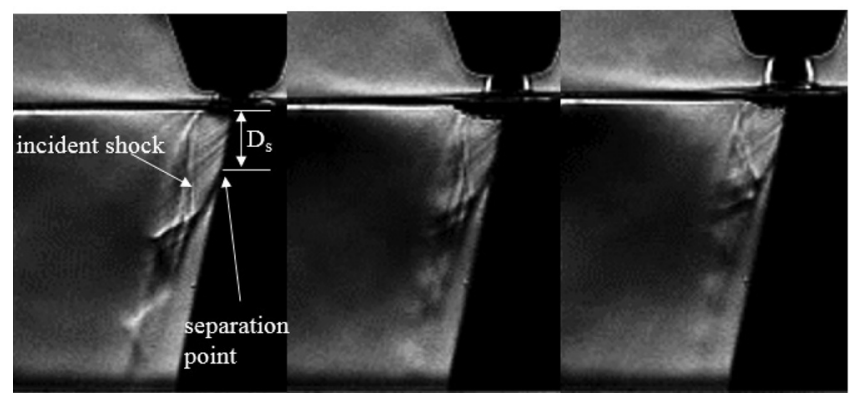

(a)

(c)

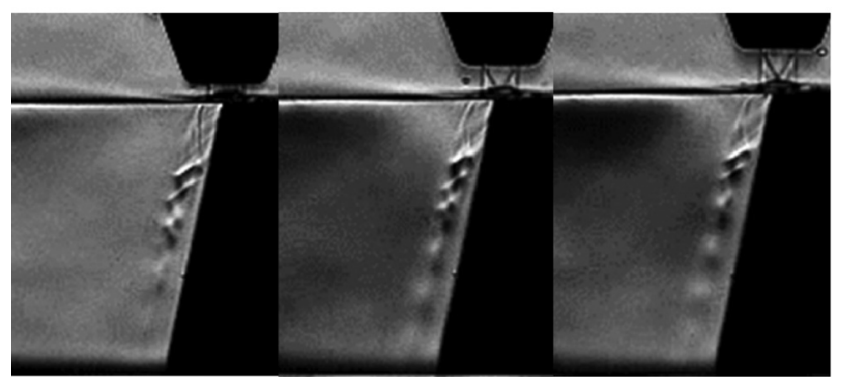

(d)

(e)

(f)

FIG. 4. Flow separation captured with different stand-off distance: (a) conical nozzle: $1 \mathrm{~mm}$, (b) conical nozzle: $2 \mathrm{~mm}$, (c) conical nozzle: $3 \mathrm{~mm}$, (d) MLN nozzle: $1 \mathrm{~mm}$, (e) MLN nozzle: $2 \mathrm{~mm}$, and (f) MLN nozzle: $3 \mathrm{~mm} . \mathrm{P}_{0}=7$ bars; kerf width $1 \mathrm{~mm}$; nozzle displacement $0 \mathrm{~mm}$; cut slope front $12^{\circ}$.

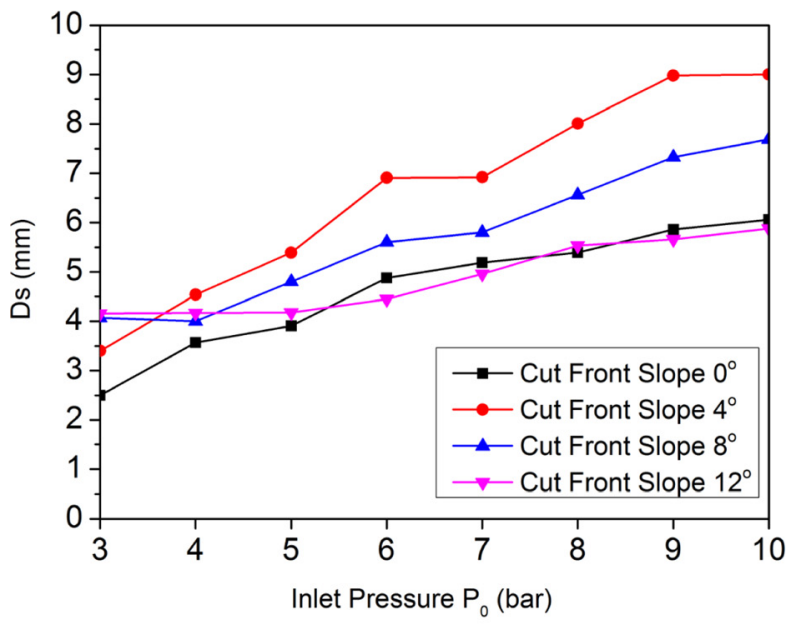

(a)

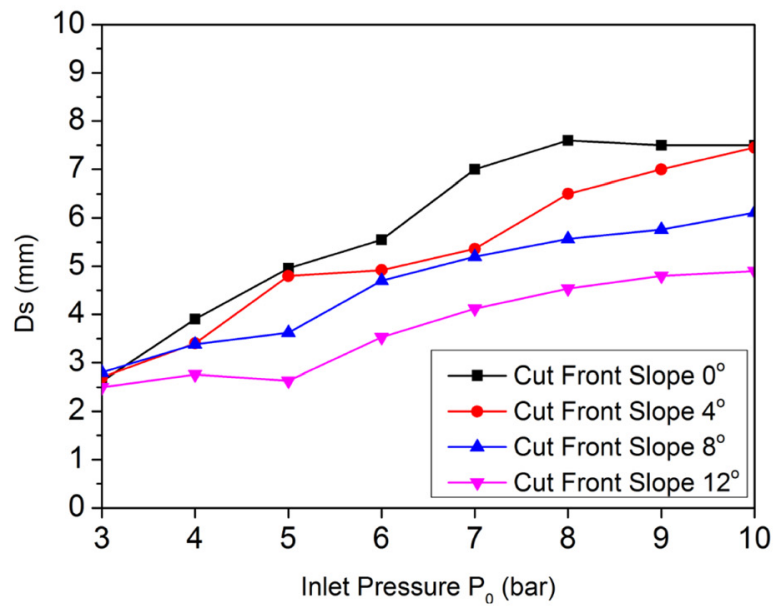

(b)

FIG. 5. Effect of inlet pressure and cut front slope on the position of flow separation point: (a) conical nozzle and (b) MLN nozzle. Kerf width $1 \mathrm{~mm}$; stand-off distance $1 \mathrm{~mm}$; nozzle displacement: $0 \mathrm{~mm}$.

slopes, $D_{\mathrm{s}}$ is close for $0^{\circ}$ and $12^{\circ}$ when the pressure changes from 4 to 10 bars. For medium slop of $4^{\circ}$ and $8^{\circ}, D_{s}$ becomes longer than that of $0^{\circ}$ and $12^{\circ}$. For the MLN nozzle in Fig. $5(\mathrm{~b}), \mathrm{D}_{\mathrm{s}}$ also increases when the inlet pressure increases from 3 to 10 bars for all the four cut front slopes. Different from the conical nozzle, Ds decreases with increasing slope angle from $0^{\circ}$ to $12^{\circ}$ under a given pressure from 4 to 10 for the MLN nozzle. For both nozzles, Ds decreases when the cut front slope increases from $4^{\circ}$ to $12^{\circ}$. The reason why Ds with MLN nozzle is longer than conical nozzle for $0^{\circ}$ under different inlet pressure is to be studied.

Figure 6 shows the effect of the inlet pressure on the position of flow separation point for conical nozzle with $1 \mathrm{~mm}$ kerf width and $4^{\circ}$ cut front. When the inlet pressure is 4,7 , and 10 bars, $D_{s}$ is $4.6,6.9$, and $9 \mathrm{~mm}$.

When the inlet pressure increases, the jet at the nozzle exit becomes more under-expanded, thus a detached shock forms near the entrance of the kerf. Some of the effects are like those in an impact jet flow. ${ }^{11}$ The higher the jet velocity before it passes through the detached shock, the lower the velocity after it passes through the shock. For the conical 


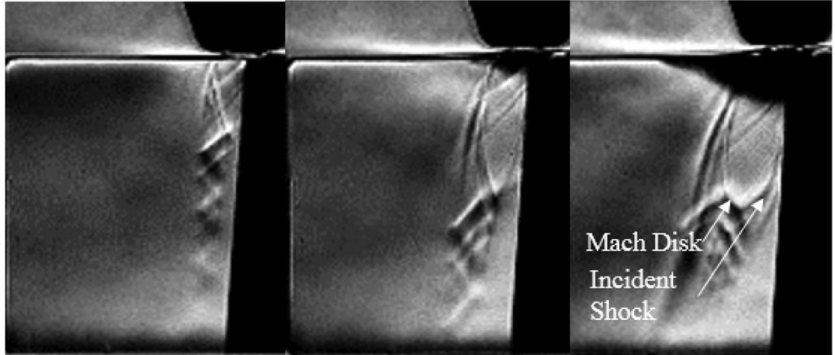

(a)

(b)

(c)

FIG. 6. Flow separation captured with different inlet pressures for conical nozzle: (a) 4 bars, (b) 7 bars, and (c) 10 bars. Cut front slope: $4^{\circ}$; kerf width $1 \mathrm{~mm}$; stand-off distance $1 \mathrm{~mm}$; nozzle displacement: $0 \mathrm{~mm}$.

nozzle when the inlet pressure is over 3 bars, the velocity of the gas jet increases with the increase of inlet pressure. Thus, the velocity of the jet entering the kerf after passing through the detached shock becomes lower with increasing the inlet pressure, and the reflect angle between the incident shock and gas boundary becomes smaller. So the position of incident shock is lower, causing $\mathrm{D}_{\mathrm{s}}$ to be bigger.

At the same time, the incident shock becomes stronger with the increase of inlet pressure because the jet entering the kerf is more under-expanded, and a Mach Disk forms with the effect of the strong incident shock and the righthand incident shock caused by the compressing effect from the vortex below the separation point at the same time, shown in Fig. 6(c). Further increase of inlet pressure would not increase $D_{s}$ as the existence of the Mach Disk will cause great energy loss of the jet. This also explains the fact that increasing the inlet pressure will not always improve the cut ability and cut quality during laser cutting of thick sections using a conical nozzle. The mechanism of the variation of the Ds with the increase of the inlet pressure using the designed MLN nozzle is similar.

Figure 7 shows the effect of cut front slope on position of flow separation point for the conical nozzle when the inlet pressure is 6 bars and kerf width is $1 \mathrm{~mm}$. $\mathrm{D}_{\mathrm{s}}$ is $6.9,5.7$, and $4.4 \mathrm{~mm}$ when the cut front slope is $4^{\circ}, 8^{\circ}$, and $12^{\circ}$. The change of cut front slope will cause the change in strength of the leading-edge shock, shown with the white arrow in Figs.

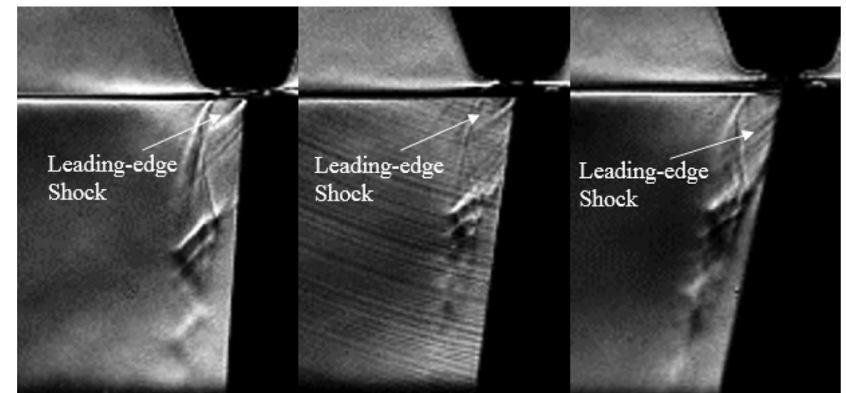

(a)

(b)

(c)

FIG. 7. Flow separation captured with different slope angles for conical nozzle: (a) $4^{\circ}$, (b) $8^{\circ}$, and (c) $12^{\circ}$. Inlet pressure: 6 bars; kerf width: $1 \mathrm{~mm}$; stand-off distance: $1 \mathrm{~mm}$; nozzle displacement: $0 \mathrm{~mm}$. 7(a)-7(c). The leading-edge shock becomes weaker with the increase of the cut front angle. The stronger the leading-edge shock, the lower the velocity of the jet passing through it. With the same nozzle and pressure, the jet enters the kerf at nearly the same velocity, and the velocity is lowest passing through the leading-edge shock when the cut front slope is $4^{\circ}$, higher when the cut front slope is $8^{\circ}$, and highest when the cut front is $12^{\circ}$. So the reflect angle between the incident shock and jet boundary is smallest when the cut front slope is $4^{\circ}$, causing the biggest $D_{s}$. The mechanism of the variation of the $D_{s}$ with the increase of cut front slope using the designed MLN nozzle is similar.

It is also noted that the designed MLN nozzle only has a small advantage over the conical nozzle as shown in Fig. 5, when the cut front is $0^{\circ}$ unlike the great advantage it has in the free jet situation. The design Mach number could be another factor affecting the flow separation point, which is to be studied.

\section{Nozzle displacement}

The effect of nozzle displacement on position of flow separation for the conical nozzle with an inlet pressure of 10 bars and a $1 \mathrm{~mm}$ kerf is shown in Figs. 8(a) and 8(b). Ds increases from 6.2 to $10 \mathrm{~mm}$ when the nozzle displacement increases from 0 to $1 \mathrm{~mm}$. When the nozzle displacement is bigger, a lot more gas enters into the kerf, and the pressure gradient of the main stream keeps positive further than the $0 \mathrm{~mm}$ displacement condition. The leading-edge shock and incident shock both become weaker at the same time, so the separation point goes down. The mechanism of the variation of the Ds with the increase of nozzle displacement using the designed MLN nozzle is similar. This can explain the fact that the focal point position should be either up or down from the surface to form a larger laser spot, to enlarge the nozzle displacement during laser cutting thick sections.

\section{Kerf width}

Effect of kerf width on position of flow separation for conical nozzle with an inlet pressure of 5 bars, a stand-off distance of $1 \mathrm{~mm}$, and a cut front angle of $0^{\circ}$ is shown in Figs. 9(a)-9(c). When the kerf width increases from 1 to

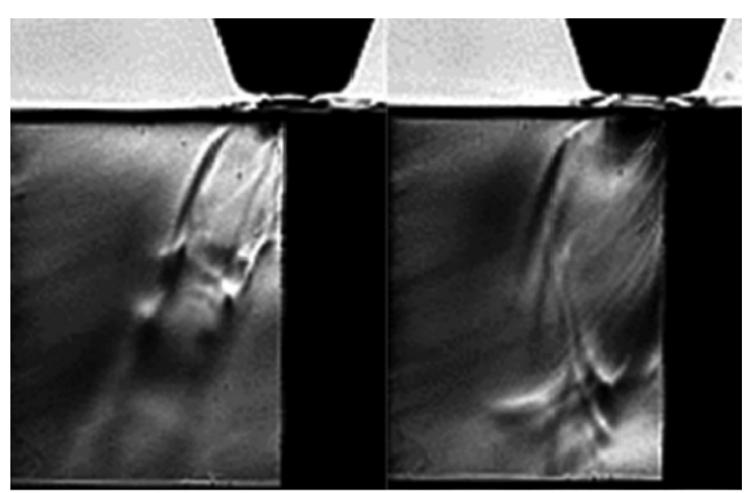

(a)

(b)

FIG. 8. Flow separation captured with different nozzle displacement for conical nozzle: (a) $0 \mathrm{~mm}$, (b) $1 \mathrm{~mm} . \mathrm{P}_{0}=10$ bars. Kerf width: $1 \mathrm{~mm}$; standoff distance: $1 \mathrm{~mm}$; cut front slope angle: $0^{\circ}$. 


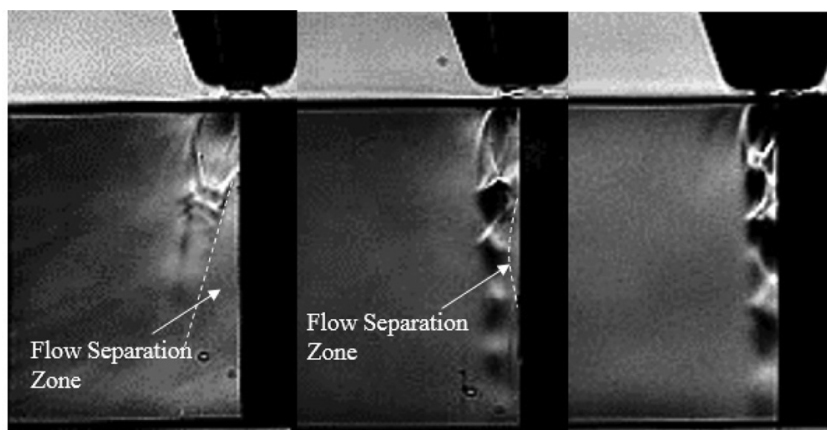

(a)

(b)

(c)

FIG. 9. Flow separation captured with different kerf width for conical nozzle: (a) $1 \mathrm{~mm}$, (b) $2 \mathrm{~mm}$, (c) $3 \mathrm{~mm}$. Inlet pressure 5 bars; cut front slope: $0^{\circ}$; stand-off distance: $1 \mathrm{~mm}$; nozzle displacement: $0 \mathrm{~mm}$.

$2 \mathrm{~mm}$ (the compress ratio, kerf width/ $\mathrm{D}_{\text {exit }}$, from 0.4 to 0.8 ), the flow separation zone becomes smaller as an arch zone shown in Fig. 9(b). When the kerf width increases to $3 \mathrm{~mm}$, the flow separation zone becomes very little. It shows that a wide cut kerf is helpful to eliminate flow separation. When the jet enters a wide kerf, the total energy loss is less since wide kerf can accommodate more jet flow. Another possible explanation is according to the wall-attachment effect based on Bernoulli Principle. When the kerf width is wider, the "pushing effect" caused by the pressure difference between the left and the right side of the jet is more significant. Thus, the "pushing effect" toward the cut front exerted on the jet weakens flow separation much more for 2 and $3 \mathrm{~mm}$ kerf width, and it is not significant for $1 \mathrm{~mm}$ kerf width.

Regarding LASOX method, laser spot is defocused and the diameter is around $3-4 \mathrm{~mm}$; the exit diameter of supersonic nozzle is around $2.5 \mathrm{~mm}$; so the cut kerf is at least $2.5 \mathrm{~mm}$ wide. It can be found that the kerf width is much wider than that of conventional laser cutting, which is generally less than $1 \mathrm{~mm}$. Flow separation zone will be very little according to the above analysis. Although wide kerf means more heat needed to melt metal, but it also can increase shear force and pressure gradient on the cut front. The latter factor is more

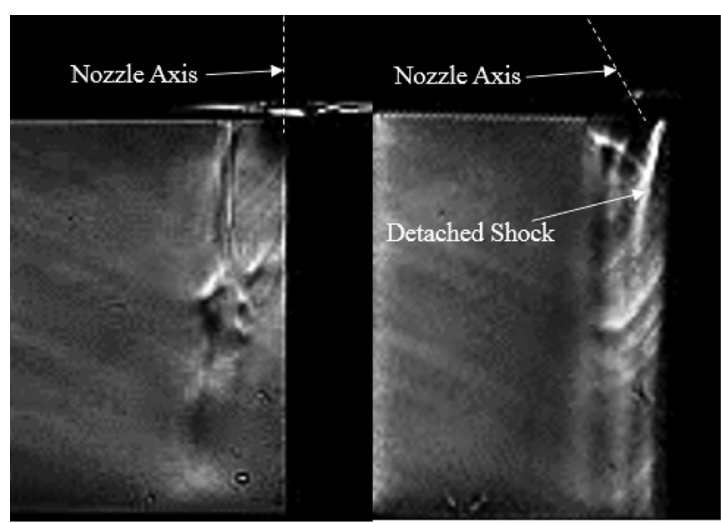

(a)

(b)

FIG. 10. Flow separation captured with different nozzle tilting angle for conical nozzle: (a) $0^{\circ}$, (b) $25^{\circ}$. Inlet pressure: 8 bars; cut front slope: $0^{\circ}$; stand-off distance: $1 \mathrm{~mm}$; nozzle displacement: $0 \mathrm{~mm}$; kerf width: $1 \mathrm{~mm}$. significant for cutting thick plates considering the removal of molten metal is a bigger issue than heat input. Therefore, LASOX makes it possible to cut thick sections up to $50 \mathrm{~mm}$ thick by suppressing flow separation inside the cut kerf.

\section{Nozzle tilting angle}

The effect of nozzle tilting angle on flow separation is shown in Fig. 10. By tilting the nozzle with an angle of $25^{\circ}$, the flow separation disappears inside the cut kerf. When the nozzle is tilted, the effect of impinging jet is much more significant. A detached shock occurs at the entrance of the kerf above the cut front, shown in Fig. 10(b). The detached shock is like a normal shock. Jet passing through the detached shock becomes highly compressed and subsonic. At the same time, the incident shock becomes much weaker and the position of the incident shock goes upper than the detached shock. The pressure gradient is positive all the way down the cut front and flow separation does not happen. However, the jet along the cut front becomes subsonic, so the shear force on the dross will be much smaller if a nozzle tilting angle is used in real cutting conditions, which is an aspect to be considered and improved.

\section{CONCLUSIONS}

Effect of stand-off distance, inlet pressure, cut front slope, nozzle displacement, kerf width, and nozzle tilting angle on flow separation was studied by Schlieren visualization for both conical and supersonic nozzles with model kerf of $20 \mathrm{~mm}$ depth.

1. The position of flow separation point goes up with increasing stand-off distance for the conical nozzle. It stays the same with changing stand-off distance for the designed MLN nozzle at its working pressure.

2. The position of flow separation point goes down with increasing the inlet pressure from 3 to 10 bars, the cut front slope from $4^{\circ}$ to $12^{\circ}$ and the nozzle displacement from 0 to $1 \mathrm{~mm}$.

3. The flow separation weakens with increasing kerf width from 1 to $3 \mathrm{~mm}$, and disappears inside the model kerf with a nozzle tilting angle of $25^{\circ}$. The above phenomena are possible strategies for controlling flow separation.

\section{ACKNOWLEDGMENT}

This work was supported by Tsinghua University Initiative Scientific Research Program.

${ }^{1}$ W. M. Steen, J. Mazumder, and K. G. Watkins, Laser Material Processing (Springer, London, UK, 2003), p. 156.

${ }^{2}$ T. Watanabe, H. Kobayashi, K. Suzuki, and S. Beppu, "Cutting of thick steel with fiber-delivered high-power Nd: YAG laser beam," Proc. SPIE 3888, 635-642 (2000).

${ }^{3}$ C. Wandera, A. Salminen, and V. Kujanpaa, "Inert gas cutting of thicksection stainless steel and medium-section aluminum using a high power fiber laser," J. Laser Appl. 21, 154-161 (2009).

${ }^{4}$ W. O'Neill and J. T. Gabzdyl, "New developments in laser-assisted oxygen cutting," Opt. Lasers Eng. 34, 355-367 (2000).

${ }^{5} \mathrm{C}$. Wandera and V. Kujanpaa, "Characterization of the melt removal rate in laser cutting of thick-section stainless steel," J. Laser Appl. 22, 62-70 (2010). 
${ }^{6}$ A. D. Brandt and G. S. Settles, "Effect of nozzle orientation on the gas dynamics of inert-gas laser cutting of mild steel," J. Laser Appl. 9, 269-277 (1997).

${ }^{7}$ H. C. Man, J. Duan, and T. M. Yue, "Analysis of the dynamic characteristics of gas flow inside a laser cut kerf under high cut-assist gas pressure," J. Phys. D: Appl. Phys. 32, 1469-1477 (1999).

${ }^{8}$ O. B. Kovalev, P. V. Yudin, and A. V. Zaitsev, "Modeling of flow separation of assist gas as applied to laser cutting of thick sheet metal," Appl. Math. Modell. 33, 3730-3745 (2009).
${ }^{9}$ G. V. Ermolaev, P. V. Yudin, F. Briand, A. V. Zaitsev, and O. B. Kovalev, "Fundamental study of $\mathrm{CO}_{2}$ - and Fiber laser cutting of industrial materials with high speed visualization technique," J. Laser Appl. 26, 042004 (2014).

${ }^{10}$ A. Matsunawa, K. Amano, and K. Nishiguchi, "Notch formation mechanism in low speed oxygen gas cutting," Trans. JWRI 10, 1-11 (1981).

${ }^{11} \mathrm{~K}$. Chen, Y. L. Yao, and V. Modi, "Gas jet-workpiece interactions in laser machining,” J. Manuf. Sci. Eng. 122, 429-438 (2000). 